
\title{
25 Research Square \\ Circular 3D Printing: Reprintable Polymers for Digital Light Processing Printing
}

\section{Guangda Zhu}

Institute of Chemistry, Chinese Academy of Sciences

Yi Hou

Beijing National Laboratory for Molecular Sciences, Institute of Chemistry, Chinese Academy of

Sciences (ICCAS)

Jian Xu

Institute of Chemistry https://orcid.org/0000-0002-9370-4829

Ning Zhao ( $\nabla$ zhaoning@iccas.ac.cn )

Institute of Chemistry, Chinese Academy of Sciences

\section{Article}

Keywords: Circular 3D Printing, Reprintable Polymers, Digital Light Processing Printing

Posted Date: July 21 st, 2020

DOI: https://doi.org/10.21203/rs.3.rs-41093/v1

License: (1) (1) This work is licensed under a Creative Commons Attribution 4.0 International License. Read Full License 
Circular 3D Printing: Reprintable Polymers for Digital Light Processing Printing

Guangda $\mathrm{Zhu}^{1,2}$, Yi Hou ${ }^{1,2}$, Jian $\mathrm{Xu}^{1,3} *$ and Ning Zhao ${ }^{1,2, *}$

${ }^{1}$ Beijing National Laboratory for Molecular Sciences, Laboratory of Polymer Physics and Chemistry, Institute of Chemistry, Chinese Academy of Sciences (ICCAS), Beijing, 100190, P. R. China.

${ }^{2}$ University of Chinese Academy of Sciences, Beijing, 100049, P. R. China.

${ }^{3}$ College of Chemistry and Environmental Engineering, Shenzhen University, Guangdong, 518060, P. R. China.

(J.X.) E-mail: jxu@iccas.ac.cn; (N.Z.) E-mail: zhaoning@iccas.ac.cn 


\begin{abstract}
3D printing has become a disruptive technology and shown great potential for various practical applications. Specially, digital light processing (DLP) demonstrates advantages in high resolution and high efficiency. However, extensive production of infusible and insoluble thermosets in DLP printing causes serious resource waste and environmental problems after disposal. Herein, we report a reprintable linear polymer for circular DLP printing. Taking advantage of the dissolution of linear polymer in its monomer, printed objects can be recycled into liquid resin and reprinted via the same DLP. Polymerization kinetics and printing resolution of recycled resins, and mechanical properties of reprinted polymers retain identical as the original. Thermoplastic nature endows 3D objects with welding and reshaping property. Fully recyclable composites have also been successfully fabricated and sustainable usage of high-value fillers comes true. This strategy helps to address environmental issues arising from unprocessable thermosets and contributes to developing a circular materials economy.
\end{abstract}




\section{Introduction}

3D printing has become a disruptive technology across electronics, aerospace, biomedical and automotive industries ${ }^{1-7}$. In particular, based on the rapid light-curing process of liquid resins, digital light processing (DLP) 3D printing shows the advantages in creating complex 3D objects with high-resolution geometry and high printing efficiency ${ }^{8-15}$. During DLP printing process, the rapid liquid-solid transition under light irradiation always requires dual-functional or multi-functional monomers, which cause 3D items to be constructed by thermosets cross-linked by covalent bonds $^{13,16-19}$. Thermosets show better durability and mechanical properties than uncross-linked thermoplastics, however, their infusible and insoluble features make them hard to be reprocessed or recycled, and will be inevitably discarded as waste at the end of their life cycle ${ }^{20-24}$. The gradually expanded DLP application and the complex 3D geometries of the products will result in more serious resources waste and environmental problems.

To address the above-mentioned problems, thermoplastics and dynamically crosslinked thermosets have been successfully developed for DLP printing ${ }^{19,25,26}$. The obtained 3D objects can be reprocessed via heating or solution processing, however, they cannot convert into liquid resins for reprinting by the same DLP, failing to recover the high-resolution geometry of original 3D objects. Although polymers with infinitely chemical recyclability have been successfully prepared ${ }^{27-30}$, these monomers lack photo-initiated reactive groups and thus cannot be used in DLP printing.

Herein, we found that mono-functional isobornyl acrylate (IBOA) can be applied in DLP printing. Different from the traditional DLP products of thermosets, the 3D objects we prepared are consisting of linear polymer of poly(IBOA). The linear 
poly(IBOA) can dissolve in IBOA monomer completely and the resultant solution can be used as a new liquid resin to reprint 3D objects by the same DLP. The transition between solid objects and liquid resin can be realized indefinitely, thus making circular DLP printing possible. The proper viscosity of liquid resin, and the rapid polymerization rate to yield linear polymer with suitable solubility in its monomer contribute to the quick transition from liquid resin to solid with high-resolution geometry. The original and recycled 3D objects show almost the same printing resolution and printing efficiency, along with comparable mechanical and thermal properties. The thermoplastic nature endows the $3 \mathrm{D}$ objects with welding and reshaping property, which can be used to improve manufacturing efficiency and overcome the size limitation by printing area. Meanwhile, fully recyclable functional composites can be prepared. After usage, the polymer matrix and the high-value functional fillers can be separated and reused. This research not only expands the range of material candidates and improves the manufacturing efficiency of DLP printing, but also contributes to a circular materials economy.

\section{Results}

Printing and reprinting process. The DLP printing and recycling process is schematically shown in Fig. 1. Patterned light illuminated from a laser projector stimulates the liquid resin to locally polymerize into a patterned solid layer with a thickness of $100 \mu \mathrm{m}$. After that, as the retracting stage moves upward, the designed 3D complex architecture will be constructed layer by layer (Fig. 1a). Here, monofunctional IBOA was used as monomer, with camphorquinone (CQ) and 4dimethylaminobenzoate (EDMAB) as the photoinitiator, and phthalocyanine Blue 15 as photoabsorber. For traditional DLP printing, bi- and/or multi-functional monomers 
are used to produce 3D objects constructed by thermosets cross-linked by covalent bonds. Differently, the proper viscosity of the printing resin and the rapid polymerization rate of this mono-functional IBOA contribute to the rapid transition from liquid monomer to 3D objects composed of linear poly(IBOA) (Fig. 1b). The printed 3D objects can dissolve in IBOA completely, thus forming a homogenous polymer solution consisting of linear poly(IBOA) and IBOA monomer (Fig. 1c). The solution can be used as a new liquid resin for the same DLP printing when photoinitiator was added. The above process can be infinitely repeated between the liquid resin and $3 \mathrm{D}$ objects, leading to $3 \mathrm{D}$ objects at the end of their life are not considered as waste but as raw materials, making the circular DLP printing possible.

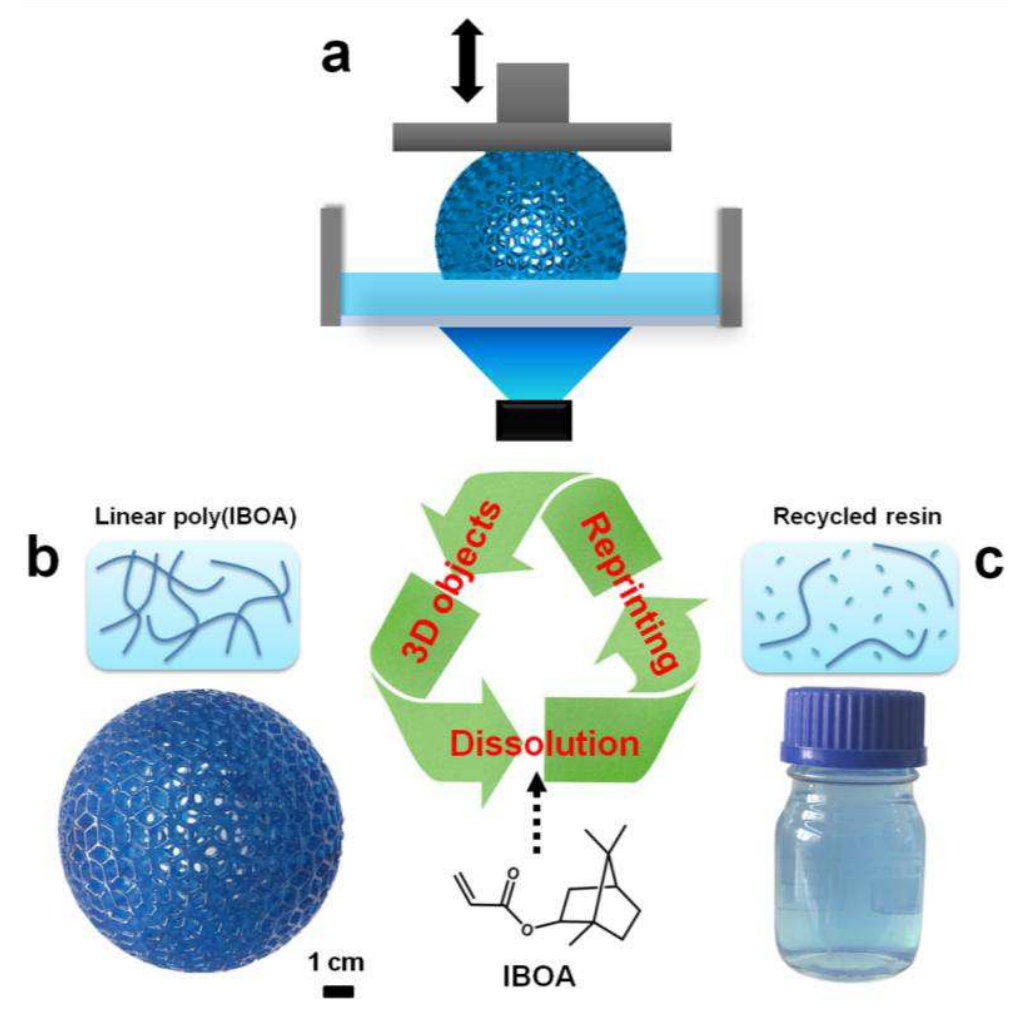

Figure 1. Circular DLP printing. (a) Scheme of DLP printing process. (b) Printed 3D objects composed of linear poly(IBOA). (c) Recycled resin of poly(IBOA) in IBOA monomer. 
Printing characteristics. Proper viscosity and rapid polymerization rate to yield a linear polymer with suitable solubility in its monomer are essential for this circular DLP printing. During DLP printing, once a layer is cured, the retracting stage will move up for the printing of next layer. A proper viscosity of the liquid resin can ensure it flows quickly and fills the gap between the cured layer and the retracting stage for the polymerization of next layer. Rapid polymerization rate can make the liquid resin quickly transform into solid, while the sufficient molecular weight of obtained linear polymer can retard its dissolution in the monomer during printing.

As Fig. 2a shows, both IBOA monomer and poly(IBOA)/IBOA solutions are transparent. The similar dynamic light scattering (DLS) results of poly(IBOA) in IBOA and in THF (a good solvent for poly(IBOA $)^{31}$ ) approved the good solubility of poly(IBOA) in IBOA (Supplementary Figure 1 and Supplementary Figure 2). IBOA has a low viscosity of $10 \mathrm{mPa} \cdot \mathrm{s}(=10 \mathrm{cps})$, while recycled solutions of poly (IBOA) in IBOA (9 wt\%) show a slightly increased viscosity of about $90 \mathrm{mPa} \cdot \mathrm{s}$ (Fig. 2b), no matter how many recycled times. The increase in viscosity results from the presence of high molecular weight poly(IBOA) in IBOA. The more poly(IBOA) added in IBOA, the higher viscosity of recycled resin. The viscosity of polymer solution at room temperature increases from 38 to $3000 \mathrm{mPa} \cdot \mathrm{s}$ with the concentration increasing from 5 to $30 \mathrm{wt} \%$ (Supplementary Figure 3). Compared with commercial photocurable resins (Supplementary Table 1), the recycled polymer solutions (5-20 wt\%) have a relatively low viscosity, particularly suitable for DLP printing, even for other rapid printing methods. ${ }^{3,32}$ Figure $2 \mathrm{c}$ shows that both IBOA monomer and recycled resins have fast and similar polymerization rates. The double bond conversion rate can reach $\sim 60 \%$ within 30 seconds, and the recycling cycles show almost no effect on the polymerization rate. 
Under light irradiation, both the original and the recycled liquid resins transform into solid films of linear poly(IBOA) with excellent transparency (Fig. 2d). The transmittance in visible range can reach around 89\% (Supplementary Figure 4). The chemical structures of virgin and recycled poly(IBOA) are consistent between rounds of recycling, as confirmed by FTIR and ${ }^{1} \mathrm{H}-\mathrm{NMR}$ (Supplementary Figure 5). The GPC results shows that all the obtained poly(IBOA)s own a molecular weight around 38 $\mathrm{kDa}$ and a similar PDI (Fig. 2e). The dissolution rate of poly(IBOA) in IBOA at static state (similar to the DLP printing process) is determined by the temperature (Fig. 2f). The dissolution rate is very low at room temperate, and the weight loss is below 3\% per hour. The weight loss of film can reach $36 \%$ per hour at $110^{\circ} \mathrm{C}$. Since the DLP printing was performed at $\sim 25^{\circ} \mathrm{C}$, the little dissolution of poly(IBOA) might not have obvious influence in DLP printing. 
a
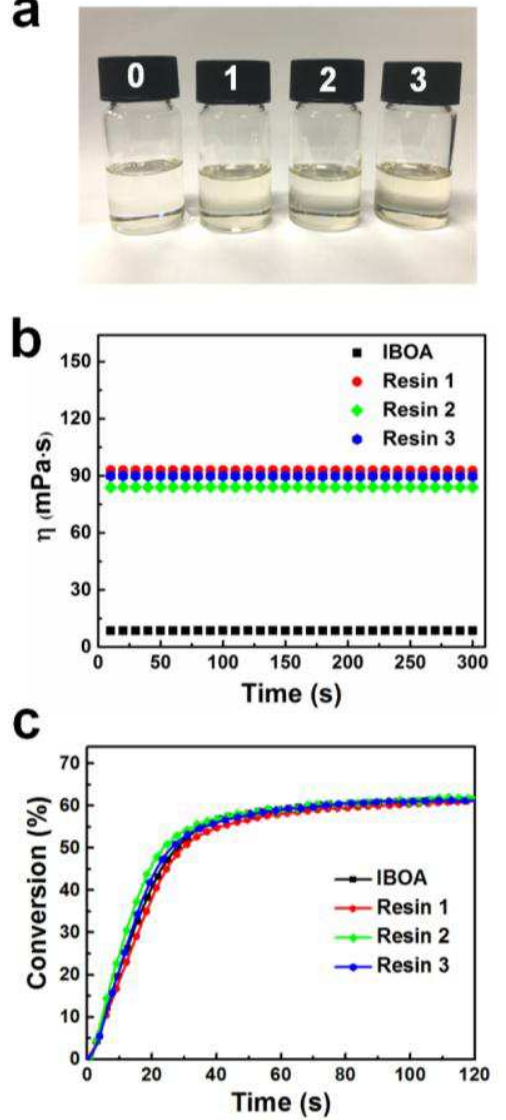

d Origin Recycle 3
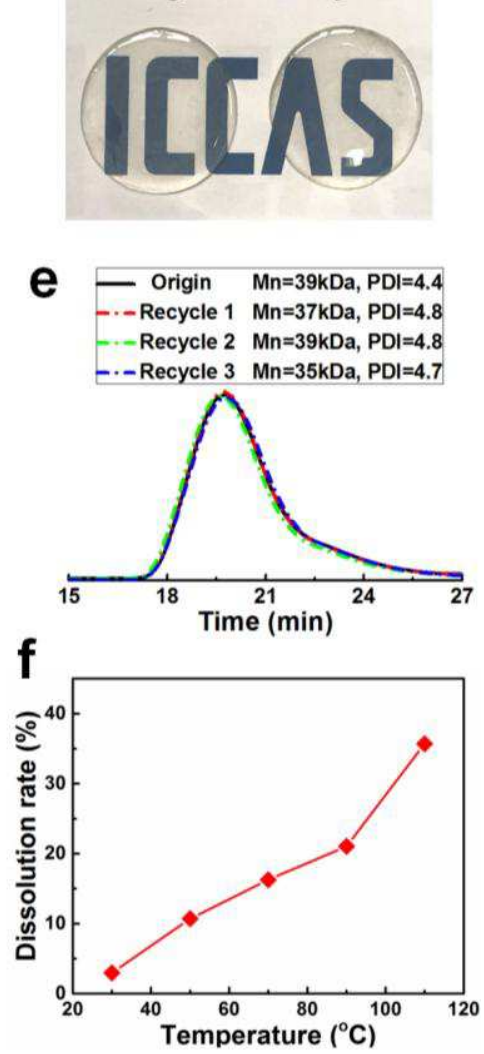

Figure 2. Printing characteristics. (a) Photography, (b) Viscosity, and (c) Reaction kinetics profiles of IBOA monomer and recycled resins of poly(IBOA) in IBOA. (d) Photography, and (e) GPC curves of original and recycled poly(IBOA)s. (f)The dissolution rate of poly(IBOA) in IBOA at different temperatures.

Reprinting performances. Fig. 3a shows models of cross rings, Chinese Bird's Nest and lattice structure printed from the pristine IBOA resin. For example, the Chinese Bird's Nest model could dissolve in IBOA monomer at $90^{\circ} \mathrm{C}$ (Fig. 3b). Almost the same models have been successfully fabricated by the same DLP method using the recycled polymer solution (Fig. 3c). In comparison, the printed commercial thermoset models could not dissolve in DMF even after 7 days (Supplementary Figure 6). Limited by the resolution of the projector, the printing resolution can reach around $300-400 \mu \mathrm{m}$. The printing resolution of 3D objects in micrometer scale can be well 
retained using the same DLP printing. By recycling utilization of materials, reduction in waste and production cost can be expected.

To verify the recyclability, mechanical and thermal properties of the original and recycled poly(IBOA)s were measured. Fig. $3 \mathrm{~d}$ shows the storage modulus and tan $\delta$ vs. temperature are consistent between rounds of recycling. Thermal gravimetric analysis (TGA) results also indicate the similar thermal stability for original and recycled poly(IBOA)s (Fig. 3e). No obvious difference in $T_{g}$ (at the peak of tan $\delta$ ) and $T_{d}$ (at the weight loss of 5\%) can be observed, no matter the recycling rounds (Supplementary Table 2). Representative stress-strain curves of the linear poly(IBOA)s before and after recycling of up to three times are shown in Fig. 3f. All samples show a comparable mechanical performance, with a tensile strength around $8.0 \mathrm{MPa}$ and a strain at break of approximately 6.5\% (Supplementary Table 2). The recycling efficiency, defined as the ratio of the mechanical properties of recycled samples to those of the original, is shown in Fig. 3g. It can be seen that the mechanical properties are largely restored after recycling. For example, the recycling efficiency of tensile strength, strain at break and Young's modulus for the sample after three recycling rounds are 102, 91 and 95\%, respectively. The water contact angles of original and recycled poly(IBOA)s changed a little, meanwhile, the printed items also exhibited a good water resistance (Supplementary Figure 7). 


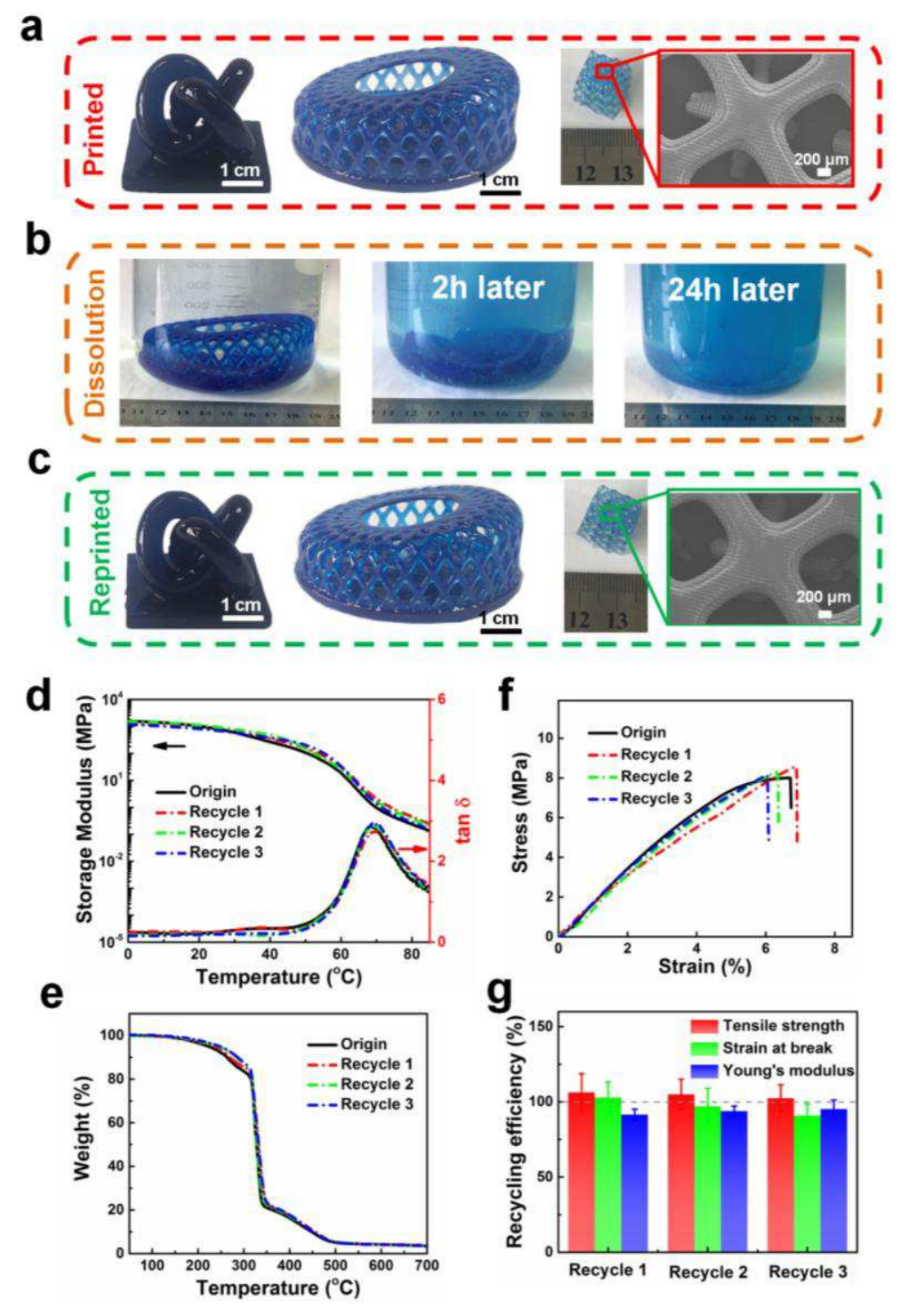

Figure 3. Reprinting performances. (a) DLP printed models using IBOA. (b) The dissolution process of printed model in IBOA. (c) DLP reprinted models using recycled poly(IBOA)/IBOA resins. (d) DMA, (e) TGA and (f) Stress-strain curves of original and recycled poly(IBOA) samples. (g) Recycling efficiency of mechanical properties for recycled poly(IBOA) samples.

Other mono-functional monomers could also be employed to realize circular DLP printing, once the requirements of proper viscosity, fast polymerization rate of the obtained linear polymer with suitable solubility in its monomer are satisfied. For example, we found that ACMO could be used, whereas methyl methacrylate and acrylic acid failed due to their slow polymerization rates. Although Zhou and Xie et al. 
reported DLP printing of ACMO, they pointed out that the obtained thermoplastics cannot be reprinted using the same DLP $\operatorname{method}^{19}$. However, we find that poly(ACMO) can dissolve in ACMO completely at a temperature above its $T_{g}$ and a recycled resin can be obtained for reprinting. The viscosity and polymerization rate of ACMO and recycled solutions of poly(ACMO) in ACMO (5 wt $\%)$, and the molecular weight of original and recycled poly $(\mathrm{ACMO})$ have been investigated (Supplementary Figure 8). 3D models have been successfully fabricated using ACMO resin and recycled resins by DLP printing (Supplementary Figure 9). DMA and mechanical tests confirmed the consistent material properties of the original and recycled samples (Supplementary Figure 10). It is worth mentioning that poly(IBOA) can dissolve in ACMO monomer and the obtained polymer solution can be reprinted to form models of poly(IBOA)/poly(ACMO) blends (Supplementary Figure 11). This not only suggests a more diverse way for material recycling, but also provide a manner to adjust the properties of products via polymer blending ${ }^{33,34}$.

Welding and reshaping. The thermoplastic nature makes printed $3 \mathrm{D}$ objects able to weld or reshape. The welding process of printed butterfly is schematically illustrated in Fig. 4a. Two printed butterflies (a red one and a blue one) were both cut into half parts. The red half and the blue half can be welded together by heating or applying some IBOA resin at the cut area under light irradiation. The welded butterfly can sustain a heavy weight of $200 \mathrm{~g}$ (Fig. 4b). Compared with the origin, both heat and IBOA welded samples can retain more than $90 \%$ mechanical properties (Supplementary Figure 12). The recovery of the mechanical properties is mainly due to the efficient reformation of polymer chain entanglement at the welding area. More importantly, complex structures can be prepared by a reshaping and welding manner, forgoing the time-consuming layer-by-layer printing process. Here, a thin film was 
printed and then transformed into a windmill at $90^{\circ} \mathrm{C}$ (higher than the $T_{g}$ of the linear polymer). The manufacturing efficiency increases by 20 times by printing a $1 \mathrm{~mm}$ thick film than directly printing the $2 \mathrm{~cm}$ high windmill (Fig. 4c). For printing larger objects, this strategy can further improve the printing efficiency and reduce the restriction on the production size due to the limited printing area.

Fully recyclable polymer composites. Introducing functional fillers into polymers can prepare composites with enhanced properties ${ }^{9,35-39}$. However, once the high-value fillers were composed into thermosets, they are hard to be recycled or reused. Herein, the reprintable nature of linear polymers makes it possible to realize recycling of polymer matrix and functional fillers from printed composites. Fig. $4 \mathrm{~d}$ schematically illustrates the recycling process. Taking CNTs as example, an object of CNTs/poly(IBOA) composite (0.5 wt\% CNTs) was printed (Fig. 4e). The object was broken into pieces and decomposed in liquid resin. The obtained dispersion was then separated into polymer solution and CNTs (Supplementary Figure 13 a). The polymer solution could be reprinted into a new 3D object of poly(IBOA) (Supplementary Figure 13 b), while the recycled CNTs showed no difference in TGA results with the original CNTs (Supplementary Figure 13 c). By adding additional CNTs, new composite resin was obtained and could be reprinted to form a new 3D object (Fig. 4f). Recycled composites containing $\mathrm{Al}_{2} \mathrm{O}_{3}$ and liquid metal were also successfully fabricated (Supplementary Figure 14). 
a
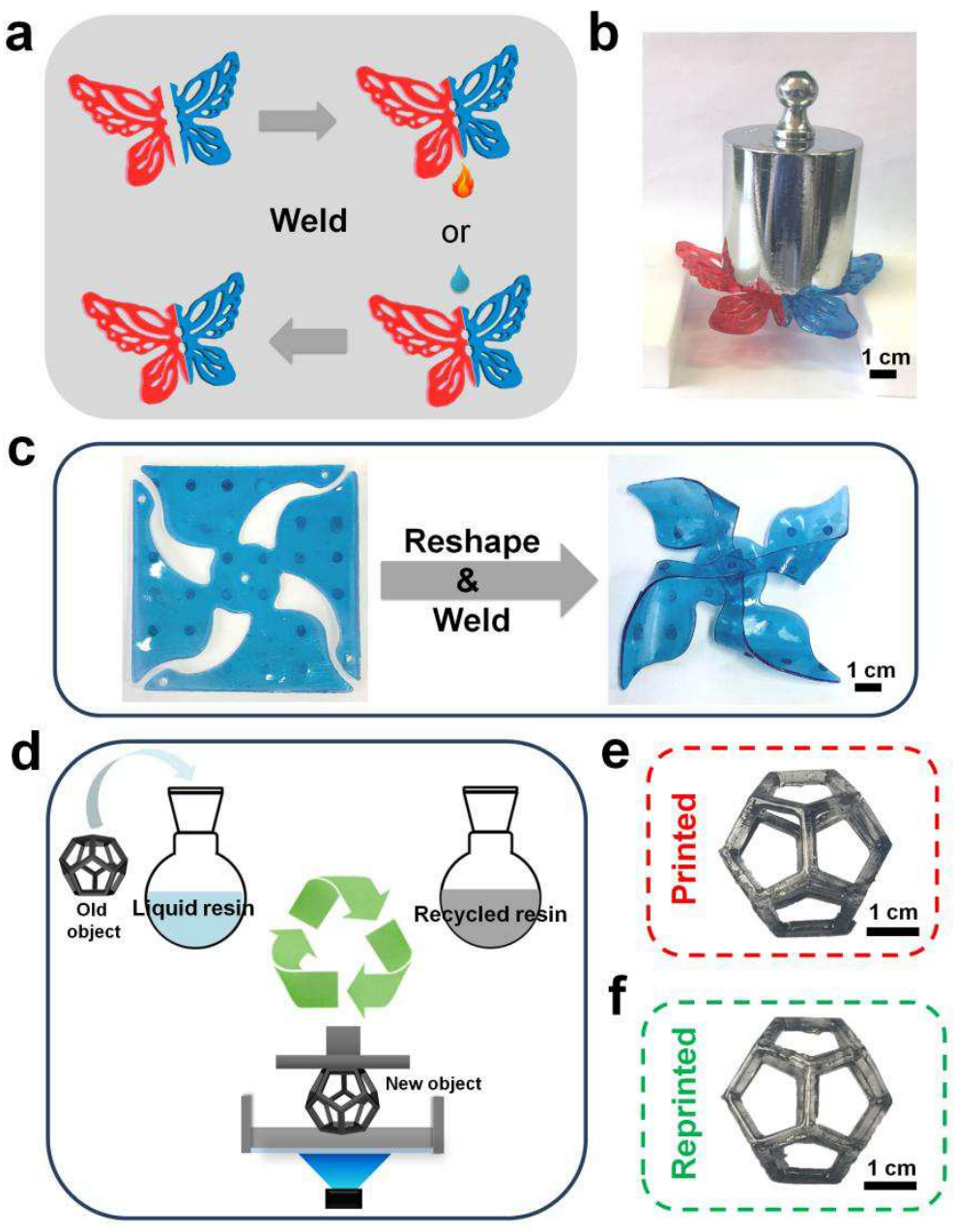

Figure 4. Welding, reshaping and fully recyclable composites. (a) Illustration of the welding process. (b) The weight-bearing test of printed butterfly after welding. (c) Printed film was reshaped into a 3D windmill. (d) Schematic illustration of the recycling process of printed composites. (e) CNTs/poly(IBOA) composite structure printed from pristine resin. (f) Reprinted CNTs/poly(IBOA) composite structure from recycled resin.

\section{Discussion}

To the best of our knowledge, it is for the first time to report recyclable thermoplastics that can be used for circular DLP printing. The printing resolution and efficiency of our thermoplastics are comparable to those of the traditional thermosetting resins. Although thermosets show a better solvent and heat resistance, the presently wide application of DLP in personalized product customization and model verification does 
not need high durability of products. On the contrary, the short service life of the products involved in these fields will result in disposal of plenty of products after use, thus wasting resources and causing great trouble in environment if thermosets were used. Therefore, developing reusable thermoplastics is of great significance for resource conservation and environmental protection.

We realized a circular DLP printing by taking advantage of the dissolution of the linear polymer in its mono-functional monomer to form a reprintable resin. The proper viscosity and rapid polymerization rate of monomer contributes to the quick transformation from liquid to solid, enabling a lay-by-layer construction of 3D complex objects via DLP printing. The recycled resin also has appropriate viscosity and polymerization kinetics, and the reprinted objects have identical printing resolution and material properties as the original. More importantly, the thermoplastic nature of linear polymer makes printed 3D objects able to weld and reshape, offering a way to increase the manufacturing efficiency and reduce the size limit subjected to the printing area. Fully recyclable composites were also successfully fabricated. This study provides an effective way to address the environmental issues arising from unprocessed thermosets produced by traditional DLP printing and make a real circular materials economy come true.

\section{Methods}

Printing resin preparation. Printing liquid resin was prepared by mixing the photoinitiator (1 g camphorquinone (CQ) and $1 \mathrm{~g}$ ethyl 4-dimethylaminobenzoate (EDMAB)) with $100 \mathrm{~g}$ isobornyl acrylate (IBOA) under stirring to form a homogenous solution. Phthalocyanine Blue 15 or oil red $\mathrm{O}$ was added as the photoabsorber $(0.02-0.5 \mathrm{wt} \%)$. The printing composite resins were prepared by 
adding carbon nanotubes $(0.5 \mathrm{wt} \%)$, gallium (3 wt $\%)$ or $\mathrm{Al}_{2} \mathrm{O}_{3}(5 \mathrm{wt} \%)$ in the above liquid resin, respectively. Other printing resin was prepared with the same method using 4-acryloylmorpholine (ACMO) as monomer.

DLP 3D printing. 3D objects were printed via a commercial DLP-based 3D printing system equipped with a blue laser projector $(445 \mathrm{~nm})$. All the models were printed with a slice thickness of $100 \mu \mathrm{m}$. After printing, the printed objects were washed by ethanol to remove the unreacted monomers.

Recycling process. The recycled printing resins were prepared by adding the printed objects $(5,10,20,30 \mathrm{~g})$ into IBOA monomer $(100 \mathrm{~g})$ under stirring at $90^{\circ} \mathrm{C}$ until transparent solutions were obtained, with a concentration of 5, 9, 17, 23 wt $\%$, respectively. Then, $1 \mathrm{~g} \mathrm{CQ}, 1 \mathrm{~g}$ EDMAB and 0.02-0.5 g phthalocyanine Blue 15 were added into the solution. Printed composite objects (10 g) were added in $100 \mathrm{~g}$ IBOA under mixing at $90^{\circ} \mathrm{C}$ until form homogeneous solution, and then the obtained solution was separated via centrifugation at $8000 \mathrm{rpm}$ for $10 \mathrm{~min}$. The separated fillers were washed by ethanol three times and dried.

Charaterizations. Nuclear magnetic resonance (NMR) spectra were recorded on a Bruker Fourier $400 \mathrm{MHz}$ spectrometer using $\mathrm{CDCl}_{3}$ as the solvent. Fourier transform infrared (FTIR) spectra were recorded on a Perkin-Elmer 2000 FTIR spectrophotometer by 32 scans from 4000 to $400 \mathrm{~cm}^{-1}$. The double bond conversion was measured using a Thermal Fisher Nicolet 5700 instrument. Light (465 nm, 10 $\mathrm{mW} / \mathrm{cm}^{2}$ ) was irradiated on liquid resin between two $\mathrm{KBr}$ plates to initiate the polymerization. The double bond conversion was calculated by the decrease of the characteristic band between 1645 and $1583 \mathrm{~cm}^{-1}$. Rheology measurements were performed on a Physical MCR 302 rheometer (Anton Paar, Austria). Viscosities of the 
printing resins were measured at a shearing rate of $50 \mathrm{~s}^{-1}$ for $5 \mathrm{~min}$ at room temperature. Gel permeation chromatography (GPC) investigation was performed on a Waters 1515 HPLC with three Styragel columns (HT3, HT4 and HT5) and 2414 differential refractive index detector. Tetrahydrofuran was used as eluent at $1 \mathrm{~mL} / \mathrm{min}$. Polystyrene standards were used to establish a calibration curve. Poly(IBOA) films $(\sim 1.5 \mathrm{~g})$ were immersed in IBOA at different temperatures and the weight of film was measured per hour to verify the dissolution rate. UV-vis transmittance spectra were obtained by using a UV-vis spectrometer (Lambda950, Perkin-Elmer). Scanning electron microscope (SEM) images were captured by a JSM-7500F (JEOL) at $5 \mathrm{~V}$ accelerating voltage. Tensile measurements were performed using a SUNS UTM4104 Instrument with a $5 \mathrm{~mm} / \mathrm{min}$ strain rate at room temperature $\left(\sim 26^{\circ} \mathrm{C}\right)$. Printed dogbone shaped samples were used. Each result was the average of the data from at least three samples. Dynamic mechanical analysis (DMA) was conducted on a DMAQ800 apparatus (TA Instrument) in a tensile film mode. Rectangular samples (ca. $10 \mathrm{~mm}$ (L) $\times 5 \mathrm{~mm}(\mathrm{~W}) \times 0.5 \mathrm{~mm}(\mathrm{~T}))$ were tested at a frequency of $1 \mathrm{~Hz}$ and a strain of $0.1 \%$. Thermogravimetric analysis (TGA) was carried out on a TA instruments Q600 Simultaneous Thermal Analyzers, at a rate of $10{ }^{\circ} \mathrm{C} / \mathrm{min}$ from 50 to $700^{\circ} \mathrm{C}$ under a nitrogen atmosphere.

\section{References}

1. Bartlett N. W., et al. A 3D-printed, functionally graded soft robot powered by combustion. Science 349, 161-165 (2015)

2. Lee A., et al. 3D bioprinting of collagen to rebuild components of the human heart. Science 365, $482-487$ (2019).

3. Walker D. A., Hedrick J. L. \& Mirkin C. A. Rapid, large-volume, thermally controlled 3D printing using a mobile liquid interface. Science 366, 360-364 (2019).

4. Ambrosi A. \& Pumera M. 3D-printing technologies for electrochemical applications. Chem. Soc. Rev. 45, 27402755 (2016). 
5. Cui H., et al. Three-dimensional printing of piezoelectric materials with designed anisotropy and directional response. Nat. Mater. 18, 234-241 (2019).

6. Gantenbein S., et al. Three-dimensional printing of hierarchical liquid-crystal-polymer structures. Nature 561, 226-230 (2018).

7. Farahani R. D., Dube M. \& Therriault D. Three-dimensional printing of multifunctional nanocomposites: Manufacturing techniques and applications. Adv. Mater. 28, 5794-5821 (2016).

8. Patel D. K., et al. Highly stretchable and UV curable elastomers for digital light processing based 3D printing. Adv. Mater. 29, 1606000 (2017).

9. Fantino E., et al. 3D printing of conductive complex structures with in situ generation of silver nanoparticles. Adv. Mater. 28, 3712-3717 (2016).

10. An H. S., et al. High-resolution 3D printing of freeform, transparent displays in ambient air. Adv. Sci. 6, 1901603 (2019).

11. $\mathrm{Mu} \mathrm{X}$., et al. Porous polymeric materials by 3D printing of photocurable resin. Mater. Horiz. 4, $442-449$ (2017).

12. Zarek M., et al. 3D printing of shape memory polymers for flexible electronic devices. Adv. Mater. 28, 44494454 (2016).

13. Kuang X., et al. Grayscale digital light processing 3D printing for highly functionally graded materials. Sci. Adv. 5, 5790 (2019).

14. Traugutt N. A., et al. Liquid-crystal-elastomer-based dissipative structures by digital light processing 3D printing. Adv. Mater. 2000797 (2020).

15. Wang K., et al. 3D printing of viscoelastic suspensions via digital light synthesis for tough nanoparticleelastomer composites. Adv. Mater. 32, 2001646 (2020).

16. De Beer M. P., et al. Rapid, continuous additive manufacturing by volumetric polymerization inhibition patterning. Sci. Adv. 5, 8723 (2019).

17. Roppolo I., et al. 3D printable light-responsive polymers. Mater. Horiz. 4, 396-401 (2017).

18. Yin X. Y., et al. Monolithic dual-material 3D printing of ionic skins with long-term performance stability. $A d v$. Funct. Mater. 29, 1904716 (2019).

19. Deng S., et al. Rapid open-air sigital light 3D printing of thermoplastic polymer. Adv. Mater. 31, 1903970 (2019).

20. Sardon H. \& Dove A. P. Plastics recycling with a difference. Science 360, 380-381 (2018).

21. Long T. E. Toward recyclable thermosets. Science 344, 706-707 (2014).

22. Rahimi A. \& García J. M. Chemical recycling of waste plastics for new materials production. Nat.Rev.Chem. 1, 46 (2017). 
23. Liu W. X., et al. Oxime-based and catalyst-free dynamic covalent polyurethanes. J. Am. Chem. Soc. 139, 86788684 (2017).

24. Liu Y., et al. Completely recyclable monomers and polycarbonate: Approach to sustainable polymers. Angew. Chem. Int. Ed. 56, 4862-4866 (2017).

25. Zhang B., et al. Reprocessable thermosets for sustainable three-dimensional printing. Nat. Commun. 9, 1831 (2018).

26. Alim M. D., et al. A photopolymerizable thermoplastic with tunable mechanical performance. Mater. Horiz. 7, 835-842 (2020).

27.Garcia J. M., et al. Recyclable, strong thermosets and organogels via paraformaldehyde condensation with diamines. Science 344, 732-735 (2014).

28. Zhu J. B., Watson E. M., Tang J. \& Chen E. Y. A synthetic polymer system with repeatable chemical recyclability. Science 360, 398-403 (2018).

29. Hong M. \& Chen E. Y. Completely recyclable biopolymers with linear and cyclic topologies via ring-opening polymerization of gamma-butyrolactone. Nat. Chem. 8, 42-49 (2016).

30. Coates G. W. \& Getzler Y. D. Y. L. Chemical recycling to monomer for an ideal, circular polymer economy. Nat. Rev. Mater. (2020).

31. Brandrup J., Immergut E.H. \& Grulke E. A. Polymer Handbook.4th ed. Wiley: New York, USA (1999).

32. Tumbleston J. R., et al. Continuous liquid interface production of 3D objects. Science 347, 1349-1352 (2015).

33. Olagoke O., Robeson L. M. \& Shaw M. T. Polymer-polymer miscibility. Academic Press, New York (1979).

34. Krause S. Polymer Blends. Academic Press, New York (1978).

35.Yan J., et al. Solution processable liquid metal nanodroplets by surface-initiated atom transfer radical polymerization. Nat. Nanotechnol. 14, 684-690 (2019).

36. Markvicka E. J., Bartlett M. D., Huang X. \& Majidi C. An autonomously electrically self-healing liquid metalelastomer composite for robust soft-matter robotics and electronics. Nat. Mater. 17, 618-624 (2018).

37. Papageorgiou D. G., Kinloch I. A. \& Young R. J. Mechanical properties of graphene and graphene-based nanocomposites. Prog. Mater. Sci. 90, 75-127 (2017).

38.Burger N., et al. Review of thermal conductivity in composites: Mechanisms, parameters and theory. Prog. Polym. Sci. 61, 1-28 (2016).

39. Punetha V. D., et al. Functionalization of carbon nanomaterials for advanced polymer nanocomposites: A comparison study between CNT and graphene. Prog. Polym. Sci. 67, 1-47 (2017). 


\section{Acknowledgement}

This work was supported by Ministry of Science and Technology of China (2016YFB1100800), Chinese Academy of Sciences (No. QYZDB-SSW-SLH025) and National Natural Science Foundation of China (No. 51733008).

\section{Author contributions}

G.-D. Z., Y. H. and N. Z. designed the experiment. G.-D. Z. performed the experiments and data analysis. G.-D. Z., Y. H., and N.Z. organized and wrote the manuscript. J. X. and N. Z. directed the project.

\section{Competing interests}

The authors declare no competing interests. 


\section{Figures}

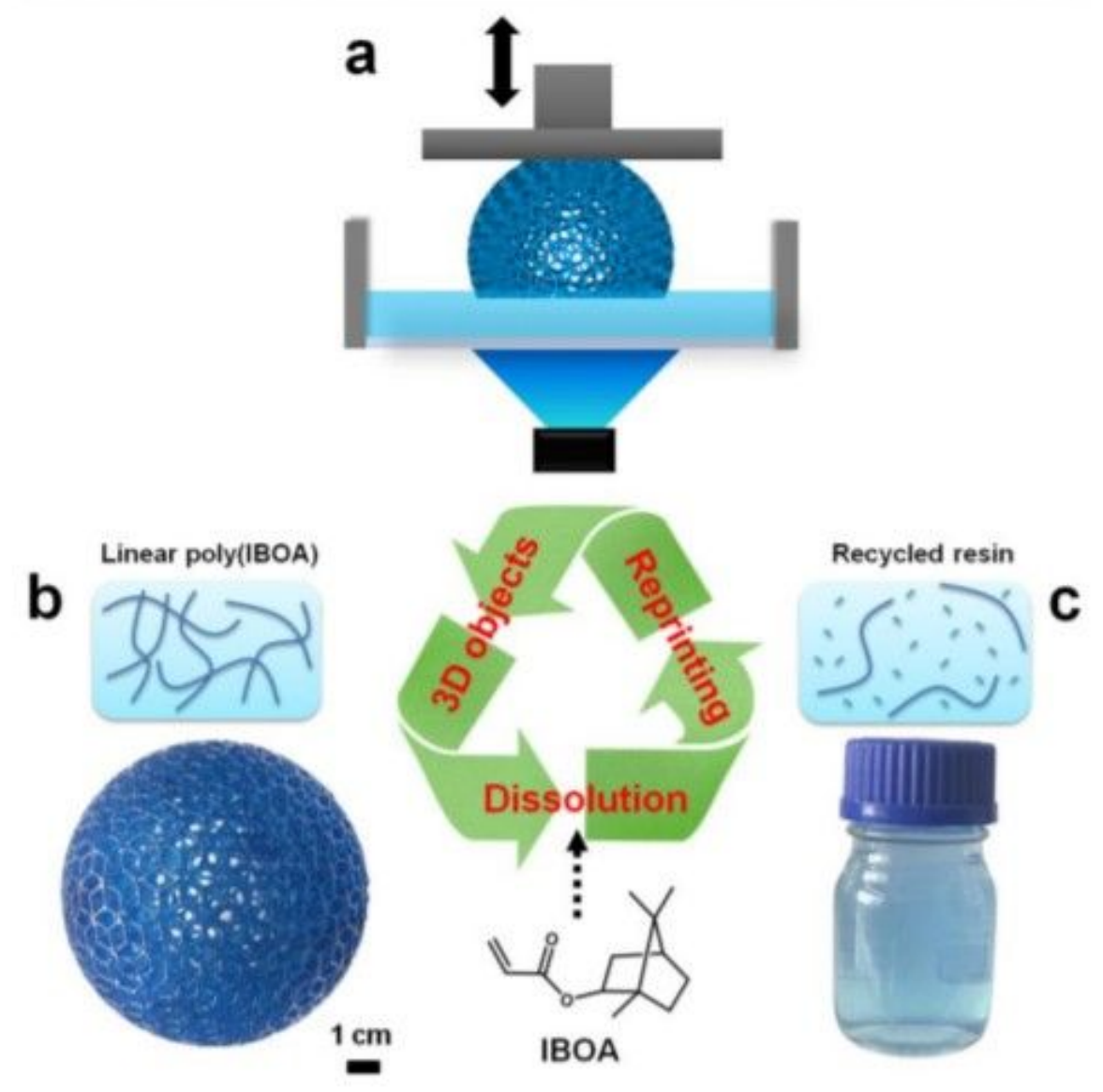

\section{Figure 1}

Circular DLP printing. (a) Scheme of DLP printing process. (b) Printed 3D objects composed of linear poly(IBOA). (c) Recycled resin of poly(IBOA) in IBOA monomer. 
a
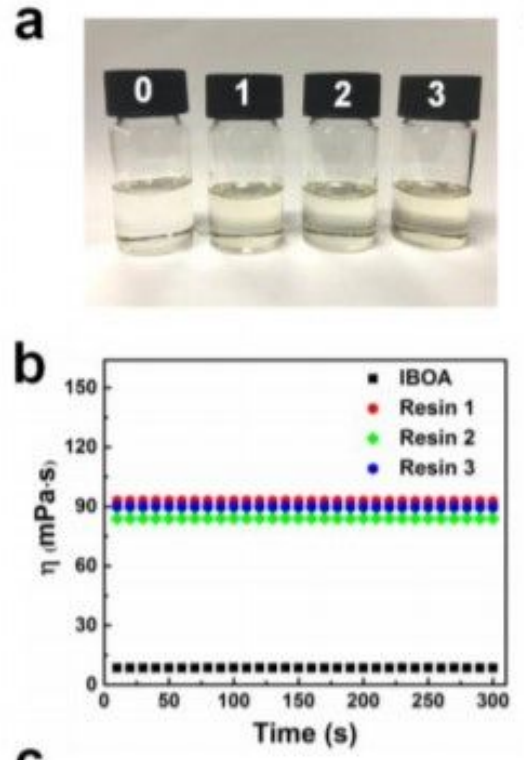

C

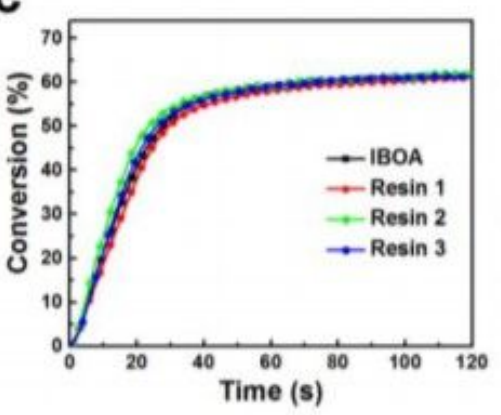

d Origin Recycle 3
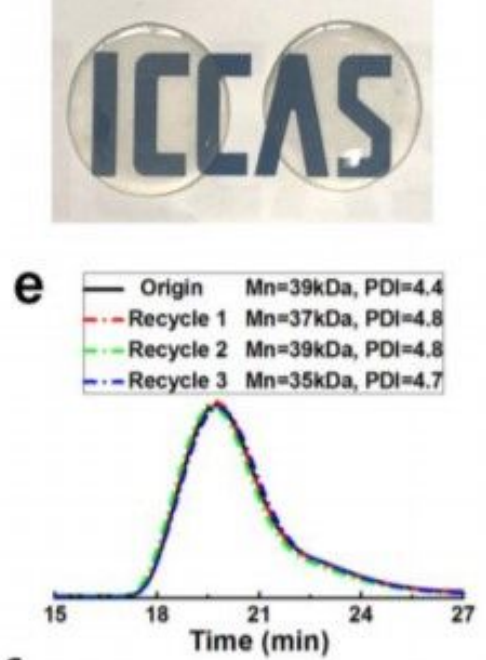

f

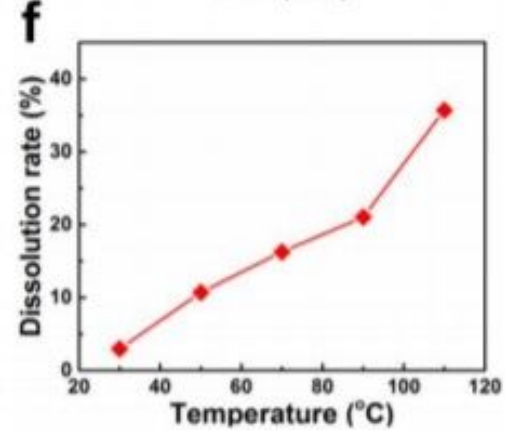

Figure 2

Printing characteristics. (a) Photography, (b) Viscosity, and (c) Reaction kinetics profiles of IBOA monomer and recycled resins of poly(IBOA) in IBOA. (d) Photography, and (e)GPC curves of original and recycled poly(IBOA)s. (f)The dissolution rate of poly(IBOA) in IBOA at different temperatures. 


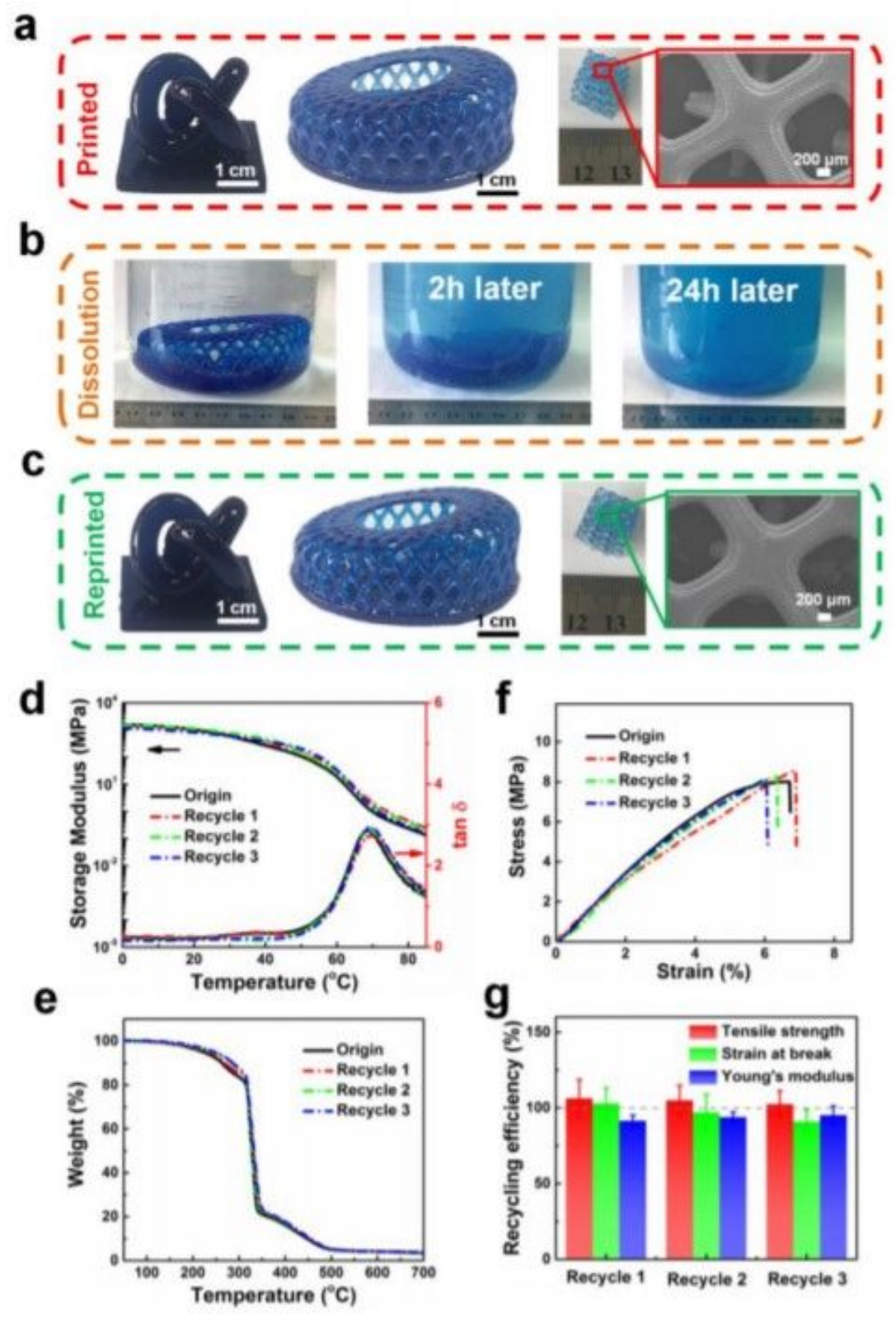

\section{Figure 3}

Reprinting performances. (a) DLP printed models using IBOA. (b) The dissolution process of printed model in IBOA. (c) DLP reprinted models using recycled poly(IBOA)/IBOA resins. (d) DMA, (e) TGA and (f) Stress-strain curves of original and recycled poly(IBOA) samples. (g) Recycling efficiency of mechanical properties for recycled poly(IBOA) samples. 
a

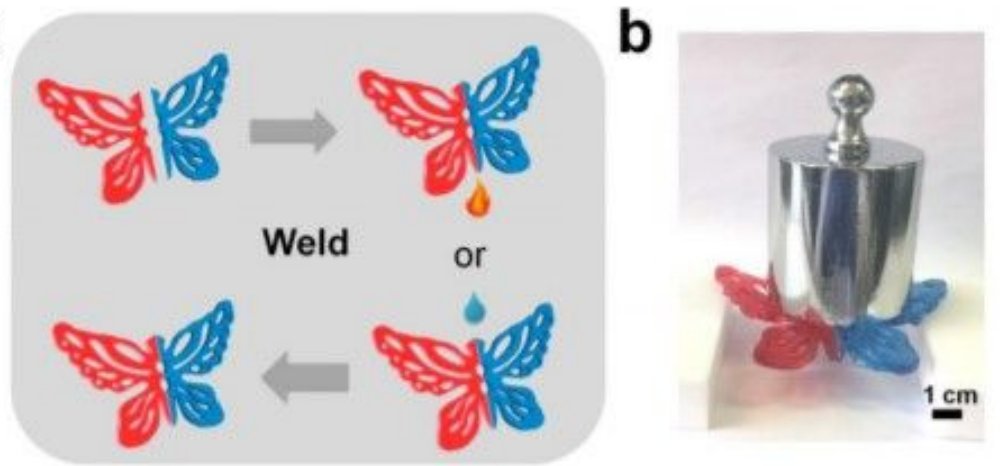

C

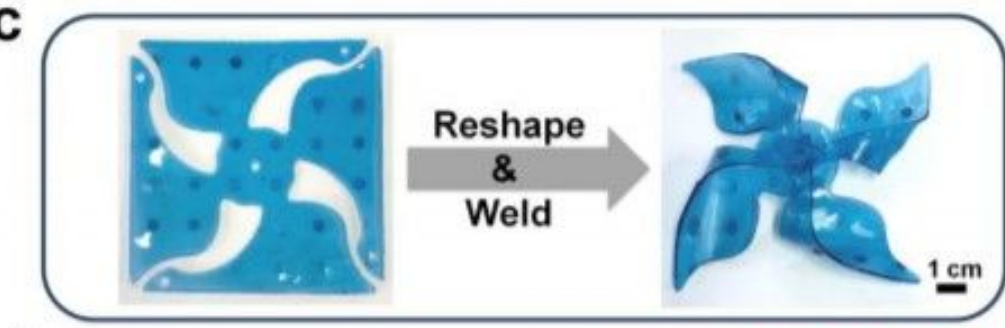

d

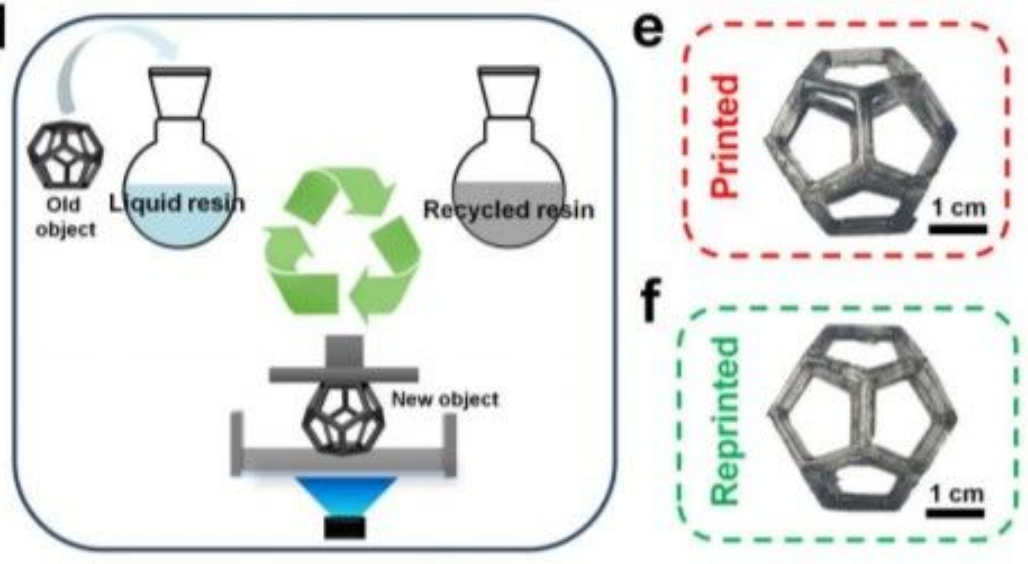

Figure 4

Welding, reshaping and fully recyclable composites. (a) Illustration of the welding process. (b) The weight-bearing test of printed butterfly after welding. (c) Printed film was reshaped into a 3D windmill. (d) Schematic illustration of the recycling process of printed composites. (e) CNTs/poly(IBOA) composite structure printed from pristine resin. (f) Reprinted CNTs/poly(IBOA) composite structure from recycled resin.

\section{Supplementary Files}

This is a list of supplementary files associated with this preprint. Click to download.

- SupportingInformation.pdf 